\title{
Linfoma No-Hodgkin y pancreatitis aguda en gestante: reporte de caso
}

\author{
Nicolás Alayón $H^{1}{ }^{1}$, José Terrón B. ${ }^{2}$, Carmen Contreras $T^{2}$, Mercedes Caba M. ${ }^{3}$, Rosa \\ Paula Pastor $V^{2}$ \\ ${ }^{1}$ Servicio de Ginecología y Obstetricia, Hospital Santa Bárbara, Puertollano, Ciudad Real. ${ }^{2}$ Unidad de Gestión Clínica \\ de Obstetricia y Ginecología, Hospital San Cecilio. ${ }^{3}$ Unidad de Gestión Clínica Provincial de Anatomía Patológica de \\ Granada. España.
}

\section{RESUMEN}

Antecedentes: El linfoma no Hodgkin durante el embarazo es una entidad rara, el tipo difuso de células grandes es aún menos frecuente y se caracteriza por una alta tasa de progresión tumoral con poca expresión clínica. Su diagnóstico y tratamiento representan un reto clínico debido a la baja incidencia de la enfermedad y a las posibles repercusiones fetales a causa del tratamiento. Caso clínico: Gestante secundípara de 31 años que ingresa por cuadro de dolor abdominal y cifras elevadas de lactato deshidrogenasa. Durante la gestación precisa varios ingresos por pancreatitis aguda de repetición y cuadro de colestasis intrahepática. Tras el parto evoluciona tórpidamente con aparición de edema en esclavina en cuello y miembros superiores, siendo diagnosticada de gran masa torácica cuya biopsia es informada como Linfoma No Hodgkin tipo B difuso de células grandes primario mediastinal. Se administran dos ciclos de tratamiento quimioterápico tras lo cual remite completamente la enfermedad. Se induce el parto con prostaglandinas intravaginal, con recién nacido de 3350 gramos y APGAR 8/10. Tras un año la paciente permanece en remisión completa.

PALABRAS CLAVE: Linfoma no-Hodgkin, linfoma difuso de células grandes, pancreatitis aguda

\section{SUMMARY}

Background: During pregnancy, Non-Hodgkin's lymphoma is a rare entity; the diffuse large cell lymphoma is still less common, and it has a high rate of tumor progression with a little clinical expression. Diagnosis and treatment is a huge challenge due to the low incidence of the condition and to the possible fetal effects because of the treatment. Clinical case: A 31-year-old woman -in her second delivery- was admitted with abdominal pain and elevated lactate dehydrogenase levels. During pregnancy, she was required several admissions reporting repeated acute pancreatitis and intrahepatic cholestasis. After delivery, it evolves into facial and upper extremity oedema, diagnosed with a large chest mass, resulting in the diagnosis of primary mediastinal large B-cell lymphoma through the biopsy. The disease goes into remission completely after two cycles of chemotherapy treatments are given. Labor is induced with intravaginal prostaglandins, with a newborn of $3350 \mathrm{~g}$ and Apgar 8/10. After a year, the patient remains in complete remission.

KEY WORDS: Non-Hodgkin's lymphoma, lymphoma diffuse large B cells, acute pancreatitis 


\section{INTRODUCCIÓN}

El linfoma difuso de células grandes (LDCG) constituye de los linfomas no Hodgkin (LNH) el tipo más frecuente. La incidencia de LNH aumenta con la edad, sin embargo el subtipo linfoma mediastinal de células grandes se caracteriza por aparecer a una edad comprendida entre los 25 y los 35 años (1).

EI LDCG típicamente describe una rápida tasa de crecimiento con sintomatología asociada al lugar de aparición de la tumoración, en más del 40\% cursa con obstrucción de la vena cava superior, lo cual es infrecuente en otros linfomas. Debido a su rápida progresión se puede diagnosticar en fases relativamente tempranas. La "sintomatología B" (pérdida de peso, fiebre y sudoración) se presenta en el $30 \%$ de los casos, en otras ocasiones aparecen astenia, disnea y adenopatías.

Para su diagnóstico las pruebas de imagen son fundamentales, pero el diagnóstico definitivo siempre lo establece la anatomía patológica, que debe ser de una pieza excisional y no de punción. Analíticamente destaca la elevación de la lactatodeshidrogenasa (LDH), expresando una medida de la carga tumoral, está elevada en el $50 \%$ de los casos al momento del diagnóstico.

La aparición de LNH durante el embarazo es rara, sin embargo es el cuarto tipo de cáncer más frecuente durante la gestación. Se estima que la incidencia es de 1-5 por cada 100.000 embarazos (2). Un estudio reciente reporta una incidencia superior de 5,39 cada 100.000 embarazos, dato que corrobora un incremento de la incidencia de este tumor (3).

El objetivo de esta comunicación es presentar un caso de linfoma no Hodgkin durante el embarazo del tipo difuso de células grandes, patología de baja frecuencia asociada a una gestación.

\section{Caso clínico}

Gestante de 31 años con un parto anterior inducido por hipertensión gestacional con elevación discreta de las transaminasas. Antecedentes familiares de leucemia y cáncer gástrico en padre y cáncer hepático en tía paterna. Como antecedentes médicos destacan hipotiroidismo en tratamiento con levotiroxina sódica, asma, dispepsia y leucopenia aislada tras el primer parto que ceden con tratamiento erradicador de Helicobacter Pylori y de forma espontánea respectivamente.

Gestación actual de evolución con normal exceptuando elevación aislada la LDH. Acude a Urgencias en semana 31+4 por dolor epigástrico de ocho horas con ligera irradiación a dorso que no cede con analgesia. Se solicita hemograma y bioquímica de control con elevación de la amilasa a $1.294 \mathrm{U} / \mathrm{L}$ y de la LDH a $1.635 \mathrm{U} / \mathrm{L}$. Ante la sospecha de pancreatitis aguda se ingresa con dieta absoluta, sueroterapia y analgesia intravenosa. Se realiza ecografía hepatobiliopancreática sin evidenciar alteraciones de interés y las cifras de amilasa y LDH descienden progresivamente sin llegar a normalizarse. Se inicia tolerancia oral pero la paciente empeora tanto clínica como analíticamente, cifras de LDH 1.432 y amilasa $1.155 \mathrm{U} / \mathrm{L}$, se decide nutrición parenteral periférica. Tras una semana continua con episodios de dolor abdominal intermitente que impiden la instauración tolerancia oral progresiva, se solicita resonancia magnética abdominal con contraste, evidenciándose bazo accesorio e imagen hipointensa en cola del páncreas de $19 \mathrm{~mm}$, sugerente de colección. En semana 33 se consigue tolerancia oral con dieta de protección biliar, pero aparece prurito corporal intenso con elevación de la bilirrubina a $1,34 \mathrm{mg} / \mathrm{dl}$ y de las transaminasas (GOT 54 y GPT 61); analíticamente destacan: LDH $1.400 \mathrm{U} / \mathrm{L}$, amilasa $474 \mathrm{U} / \mathrm{L}$ y ácidos biliares en rango normal.

Se diagnostica colestasis intrahepática y se decide tratamiento con ácido ursodesoxicólico 300 $\mathrm{mg}$, polaramine $6 \mathrm{mg}$ y valium $10 \mathrm{mg}$; también se instaura maduración pulmonar fetal ante posible interrupción precoz del embarazo. El cuadro evoluciona favorablemente aunque persiste la elevación de la LDH, se decide alta con tratamiento oral y controles seriados analíticos y ecográficos de forma ambulatoria.

En semana $35+2$ acude de nuevo a Urgencias por dolor punzante en epigastrio irradiado hacia flancos y región lumbar y persistencia del prurito que no cede con tratamiento pautado, se solicita analítica con LDH 2.006,4 U/L y GPT $56 \mathrm{U} / \mathrm{L}$, se decide ingreso hospitalario donde se realizan analíticas seriadas persistiendo la LDH elevada y la clínica descrita, sin llegar a precisar nutrición parenteral. Dada la tórpida evolución se induce el parto con prostaglandinas intravaginales finalizando espontáneamente con recién nacido femenino de 3.350 gramos y APGAR 8/10.

Persisten las molestias en epigastrio así como en región lumbar y el prurito mejora considerablemente. Se solicita ecografia abdominal con vesícula biliar hidrópica con barro biliar y microlitiasis, páncreas heterogéneo aumentado de tamaño y desestructurado y derrame pleural derecho. En los estudios analíticos destaca la LDH a 3.019,1 U/L y amilasa de $121 \mathrm{U} / \mathrm{L}$. Se diagnostica cólico biliar complicado y nueva pancreatitis aguda. Aparición de edema en esclavina en miembros superiores y en cuello. TAC toracoabdominopélvico con contraste que informa de pancreatitis aguda edematosa con necrosis de más del $50 \%$, además de trombo en aurícula derecha con reflujo del contraste a cava superior y gran masa mediastínica infiltrando vena cava superior, dificultando el flujo a ese nivel con adenopatías mediastínicas bilaterales infraclaviculares, hiliares bilaterales, carinales, en 
mediastino anterosuperior, afectación pericárdica y derrame pleural derecho (Figura 1). Se pauta enoxaparina de $60 \mathrm{mg}$ cada 12 horas, nutrición nasoenteral peptídica, dexametasona $4 \mathrm{mg}$ y se biopsia con aguja gruesa de la masa. Se produce mejoría clínica de la paciente que permite dieta progresiva de protección pancreática. Anatomía Patológica informa de Linfoma No Hodgkin tipo
$B$ difuso de células grandes primario mediastinal, CD 20 positivo, estadio II B (Figura 2). En biopsia de médula ósea hay negatividad para linfoma y se inicia quimioterapia (QT): rituximab, ciclofosfamida, adriamicina, vincristina y prednisona (CHOP-R) Tras tres días de QT aparece disnea intensa precisando pleurocentesis evacuadora derecha.

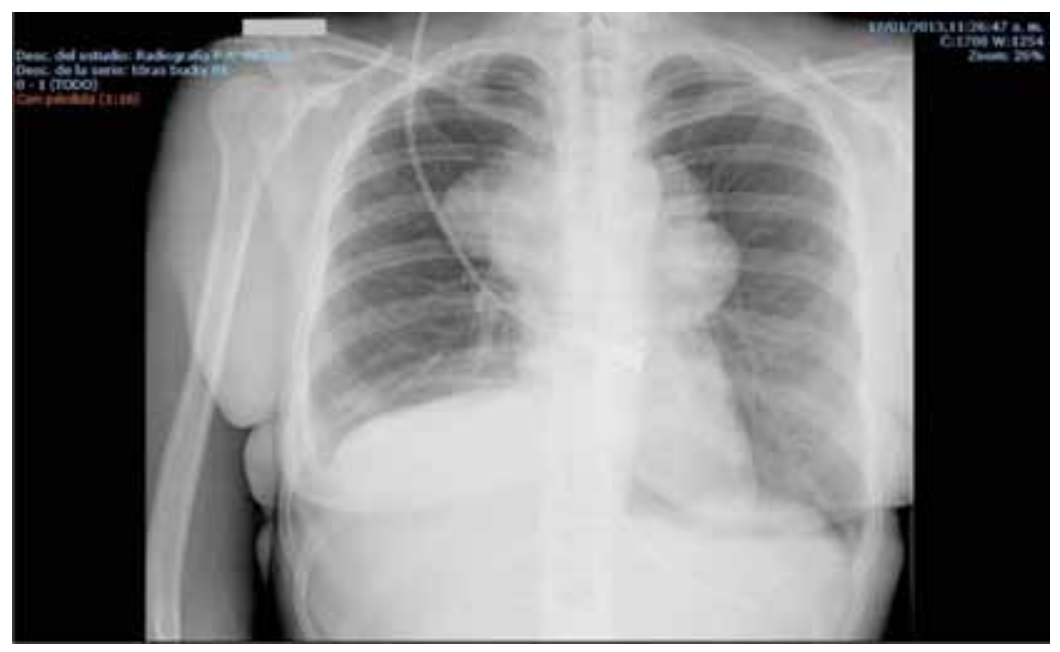

Figura 1. Masa mediastínica en el momento de su diagnóstico.

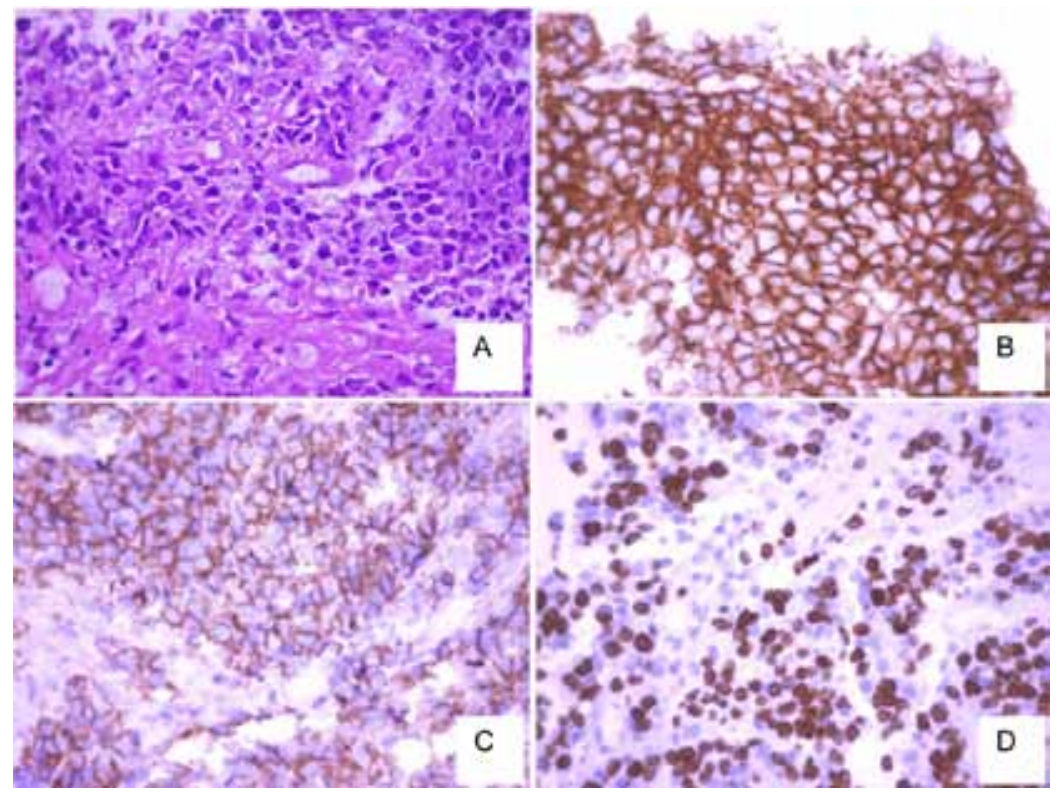

Figura 2. Neoplasia linfoide formada por células de gran tamaño, HE x60 (A). Las células tumorales son positivas para CD45, Inmunoperoxidasa x60 (B) y para marcadores de linfocitos B (CD20), inmunoperoxidasa x60 (C). El índice proliferativo, medido con la tinción para Ki67, es elevado. Inmunoperoxidasa x60 (D). 
Tras dos ciclos de QT, en tomografía por emisión de positrones (PET) aparecen varios focos hipermetabólicos prevasculares, peribronquiales y subcarinales. Se continúa con QT hasta completar seis ciclos con nueva reevaluación mediante PET persistiendo algunos focos hipermetabólicos. Se decide tratamiento quimioterápico de segunda línea ifosfamida, mitoxantrona y etopósido (MINE) durante dos ciclos y posterior auto/alo transplante de médula ósea. El estudio de histocompatibilidad HLA de la hermana de la paciente resulta compatible. Se inicia QT produciéndose respuesta completa metabólica con posterior transplante autólogo de progenitores hematopoyéticos. Tras un año con controles seriados la paciente continúa en remisión completa (Figura 3).

\section{DISCUSIÓN}

El diagnóstico de $\mathrm{LNH}$, por infrecuente de la patología durante el embarazo, representa un reto clínico, sin embargo en los últimos años se ha producido un aumento de la incidencia a nivel gestacional dado el retraso de la edad de inicio de procrear en los países desarrollados y el aumento de la tasa de VIH que se relaciona con la aparición de esta enfermedad (4). Pero en el caso específico del subtipo linfoma mediastinal de células grandes el rango de edad, a diferencia del resto de $\mathrm{LNH}$, se caracteriza por ser más temprano, como ya se ha descrito anteriormente.

Los LNH durante el embarazo son difíciles de diagnosticar, en este caso la ausencia de sintomatología asociada al lugar de la tumoración y lo infrecuente de la enfermedad durante el embarazo retrasaron el diagnóstico a después del parto. Las cifras de LDH elevadas de forma prolongada deben llamar la atención del profesional ante la sospecha de un posible proceso tumoral, aunque hay que tener en cuenta que la LDH es un parámetro analítico inespecífico que también podría estar relacionado con la pancreatitis.

En el caso presentado destaca la aparición de episodios repetidos de pancreatitis aguda. La aparición de esta enfermedad asociada al LNH sin afectación tumoral de la glándula de forma local - a distancia es extremadamente rara. Podría tratarse de un evento aislado y sin relación con el LNH. Se describen algunos casos de aparición de pancreatitis asociada a LNH sin afectación tumoral pancreática $(5,6)$. Takahashi y cols $(7)$, atribuyen una posible causa inmune a la aparición de algunas pancreatitis en el contexto del LNH denominando el cuadro clínico "pancreatitis autoinmune". Sin embargo lo infrecuente de esta asociación hace muy difícil dar una respuesta. Sí se ha demostrado que la presencia de LNH durante el embarazo aumenta el riesgo de aparición de preclampsia (3),

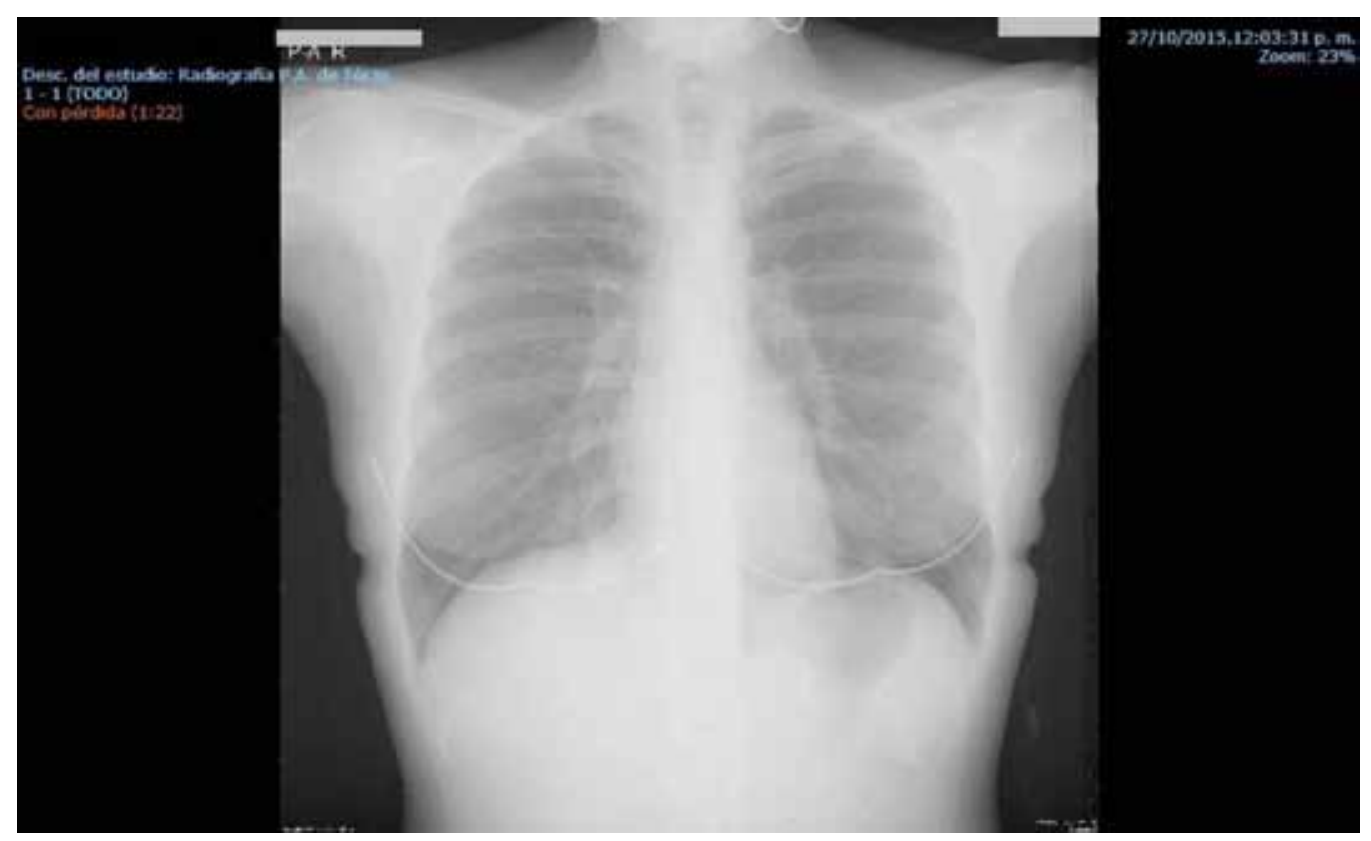

Figura 3. Remisión completa de la tumoración tras tratamiento. 
en este caso las tensiones arteriales se mantuvieron dentro de la normalidad, pero hay antecedentes de hipertensión arterial en el embarazo anterior.

Se ha sugerido una progresión más rápida y agresiva del LNH durante el embarazo, sin embargo no existen estudios que lo demuestren y en muchos casos los subtipos de LNH descritos eran variedades más agresivas por sí mismas que por el propio embarazo (8).

Si el diagnóstico durante el embarazo es difícil, su tratamiento representa un reto. La quimioterapia es la principal arma terapéutica. Tiene especial relevancia el momento gestacional del diagnóstico, dado el potencial efecto teratógeno de la quimioterapia sobre el feto.

La interrupción legal de la gestación es una opción, principalmente por debajo de las 22 semanas; además puede recomendarse en casos de LNH agresivo estadios III-IV en primer trimestre. Durante el segundo trimestre la quimioterapia se asocian a crecimiento intrauterino restringido, parto prematuro, muerte fetal, desarrollo funcional alterado, retraso mental y disminución de la capacidad de aprendizaje.

Con respecto al pronóstico de la enfermedad, concretamente en el caso del Linfoma No Hodgkin tipo B difuso, de células grandes, primario mediastinal, diagnosticado en estadios precoces y una respuesta completa al tratamiento, el riesgo de recurrencia después de un año es bajo (1).

\section{CONCLUSIÓN}

El Linfoma No Hodgkin es una entidad rara durante el embarazo, sin embargo es el cuarto cáncer más frecuente asociado al embarazo. Su diagnóstico y tratamiento durante el embarazo es un reto para la medicina materno-fetal.

\section{REFERENCIAS}

1. Murrieta González H, Villalobos Prieto A, García Correa SE. Linfoma: aspectos clínicos y de imagen. Anal Radiol Mex 2009; I:81-97.

2. Kuzel TB, Benson AB. Non-Hodgkin's lymphoma. In. Gleicher N (ed). Principles and practice of Medical Therapy in pregnancy. East Norwalk: Appleton \& Lange, 1992; 1077-81.

3. El-Messidi A, Patenaude V, Abenhaim HA. Incidence and outcomes of women with non-Hodgkin's lymphoma in pregnancy: A population-based study on 7.9 million births. J Obstet Gynaecol Res 2015;41(4):582-9.

4. Pereg $D$, Koren $G$, Lishner $M$. The treatment of Hodgkin's and non Hodgkin's lymphoma in pregnancy. Haematologica 2007;92:1230-7.

5. Huo L, Luo Y, Zhang T, Zhu Z, Li F, Zhao Y. Unexpected primary osseous lymphoma as the cause of lactic acidosis in a patient suffering from pancreatitis. Clin Nucl Med 2010;35(10):790-3.

6. Mansour $\mathrm{P}$, Adams RD, Isaacs $\mathrm{P}$, Ridway JC, Flanagan NG, Harrison CJ. T cell non-Hodgkin's lymphoma with uveitis, pancreatitis, digital gangrene and multiple chromosomal abnormalities. J Clin Pathol 1990;43:694-7.

7. Takahashi N, Ghazale AH, Smyrk TC, Mandrekar JN, Chari ST. Possible association between IgG4-associated systemic disease with or without autoimmune pancreatitis and non-Hodgkin lymphoma. Pancreas 2009;38(5):523-6.

8. Horovitz NA, Benyamini N, Wohlfart K, Brenner B, Avivi I. Reproductive organ involvement in nonHodgkin lymphoma during pregnancy: a systematic review. Lancet Oncol 2013;14:e275-e282. 